\title{
MicroRNA-30b Regulates High Phosphorus Level-Induced Autophagy in Vascular Smooth Muscle Cells by Targeting BECN1
}

\author{
Jian Wang ${ }^{a}$ Yi-Ting Sun ${ }^{b}$ Tian-Hua Xu $u^{\text {a }}$ Wei Sun ${ }^{c}$ Bin-Yao Tian ${ }^{a}$ Zi-Tong Sheng ${ }^{a}$ \\ Li Sun ${ }^{a}$ Lin-Lin Liua Jian-Fei Maa Li-Ning Wanga ${ }^{a}$ Li Yao $^{a}$
}

aDepartment of Nephrology, The First Hospital of China Medical University, ShenYang, LiaoNing, ${ }^{b}$ Department of Clinical Medicine, China Medical University, ShenYang, LiaoNing, 'Department of General Surgery, Shengjing Hospital of China Medical University, ShenYang, LiaoNing, China

\section{Key Words}

Vascular smooth muscle cells $•$ Autophagy $\bullet$ miRNAs $\bullet$ miR-30b

\begin{abstract}
Background/Aims: Autophagy is an evolutionarily conserved mechanism that affects the survival and functions of vascular smooth muscle cells (VSMCs). We explored the role of microRNAs (miRNAs) in regulating autophagy in VSMCs exposed to high phosphorus ( $\mathrm{Pi}$ ) levels. Methods: VSMCs were isolated from the thoracic aorta of rats and were cultured primarily. Real-time PCR was used to measure the mRNA expression of indicated genes. Western blotting was performed to detect the protein expression of autophagy-related markers. Results: We found that treatment with high Pi levels ( 1 and $3 \mathrm{mM}$ ) activated LC3II expression and promoted autophagic flux in VSMCs. Conversely, treatment with an autophagy inhibitor decreased LC3II expression. Pi stimulation dysregulated the expression of several miRNAs such as miR-18a, miR-21, miR-23a, miR-30b, and miR-31a. However, miR-30b overexpression decreased Pi-induced expression of autophagy-related marker genes such as BECN1, ATG5, and $L C 3 b$, whereas miR-30b downregulation increased $\mathrm{Pi}$-induced expression of these genes. In addition, we found that miR-30b directly targeted BECN1. Conclusions: These data suggest that miR-30b plays an important role in the regulation of high Pi level-induced autophagy in VSMCs by targeting BECN1.

(c) 2017 The Author(s)

Published by S. Karger AG, Basel
\end{abstract}

\section{Introduction}

Autophagy is a basally active mechanism that protects cells against the accumulation of misfolded or damaged organelles and protein aggregates. Three autophagy types have been identified, namely, microautophagy (termed as autophagy), chaperone-mediated autophagy, and macroautophagy [1, 2]. Emerging evidence indicates that autophagy plays a critical J. Wang and Y.-T. Sun are the co-first author. 


\section{Cellular Physiology Cell Physiol Biochem 2017;42:530-536

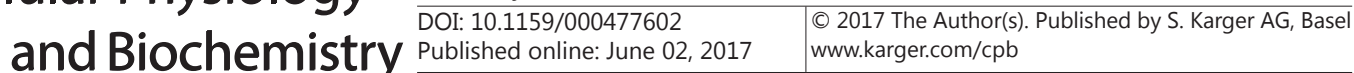

Wang et al.: miR-30b Regulates Autophagy in VSMC via BECN1

role in the maintenance of cellular homeostasis in response to environmental stimuli and nutrient deprivation $[3,4]$.

Vascular smooth muscle cells (VSMCs) play an essential role in maintaining the physiological functions of blood vessel walls. Altered VSMC function in response to intracellular and/or extracellular stimuli such as high phosphorus (Pi) levels is associated with the development of vascular diseases $[5,6]$. Autophagy regulates VSMC functions, including growth, migration, contraction/relaxation, and differentiation [7, 8]. A recent study reported that treatment with high Pi levels induced VSMC calcification, decreased VSMC marker expression, and increased osteogenic marker levels, suggesting a close relationship between Pi levels and VSMC function [5].

MiRNAs are small, noncoding regulatory RNAs that play significant roles in VSMC proliferation, development, and differentiation [9, 10]. Several studies have reported novel roles of miRNAs in autophagy regulation $[11,12]$. For instance, miR-20a and miR$106 \mathrm{~b}$ regulate leucine deprivation-induced autophagy in myoblasts by downregulating ULK1 expression [13]. Further, miR-374a and miR-630 inhibit the expression of UVRAG, a regulator of class III PI3K complex, thus regulating autophagic signaling [14]. Ding et al were the first to report that hsa-let-7 is a critical regulator of autophagy and apoptosis in VSMCs [15]. However, the role of miRNAs in VSMC autophagy has not been completely elucidated to date. Therefore, the present study explored the role of miRNAs in the autophagy of VSMCs exposed to high Pi levels.

\section{Materials and Methods}

\section{Cell culture}

VSMCs were isolated from the descending thoracic aorta of rats by performing enzymatic digestion, as described previously [15]. The cells were grown in monolayers in DMEM supplemented with 10\% FBS (Invitrogen, Carlsbad, CA), $100 \mathrm{U} / \mathrm{mL}$ penicillin, $0.1 \mathrm{mg} / \mathrm{mL}$ streptomycin, $20 \mathrm{mM}$ Tricine buffer, and 1\% non-essential amino acid solution at $37^{\circ} \mathrm{C}$ in a humidified atmosphere of $\mathrm{CO}_{2}$. Purity of the cells was checked using an immunofluorescence technique with $\alpha$-actin, as described previously [14]. Cells obtained after the sixth and tenth passages were used in the study. 293T cells were cultured in standard DMEM.

\section{Real-time PCR}

Total RNA was isolated using Trizol reagent, and $1 \mu \mathrm{g}$ RNA was reverse transcribed into cDNA. Realtime PCR was performed in a $20-\mu \mathrm{L}$ reaction mixture. $U 6$ or beta-actin gene was used as an internal control, and expression levels were calculated using $2^{-\Delta \Delta \mathrm{Ct}}$ method.

\section{Western blotting}

Cells were harvested and were lysed in lysis buffer on ice for $20 \mathrm{~min}$. Proteins extracted were electrophoresed by performing sodium dodecyl sulfate-polyacrylamide gel electrophoresis on a $12 \%$ gel. The resolved proteins were transferred onto a PVDF membrane. The membrane was incubated overnight at $4^{\circ} \mathrm{C}$ with primary antibodies against LC3I/II, beta-actin, and BECN1. After washing, the membrane was incubated with horseradish peroxidase-conjugated secondary antibodies for $1 \mathrm{~h}$ at room temperature. Protein bands obtained were detected using an enhanced chemiluminescence kit (Amersham Biosciences Co., Piscataway, NJ).

\section{Fluorescence microscopy}

VSMCs were infected with a tandem fluorescent monomeric red fluorescent protein-green fluorescent protein-tagged LC3 virus. Next, the infected cells were treated with different doses of Pi and were viewed under a fluorescence microscope (DMI6000; Leica, Solms, Germany).

\section{Dual-luciferase assay}

BECN1 3'-UTR target site was amplified by performing PCR and was cloned downstream of the Renilla luciferase gene. VSMCs were cultured in six-well plates and were cotransfected with recombinant plasmids together with miR-30b mimics or a scrambled miRNA by using Lipofectamine 2000 (Invitrogen). The cells were lysed, and luciferase activity was determined using a dual-luciferase assay kit (Promega, Madison, WI). 
Statistical analysis

All quantitative data are presented as mean \pm SE. Unpaired Student's $t$-test was used to compare two independent groups. Results were considered statistically significant at $P<0.05$.

\section{Results}

High Pi levels induce autophagy in VSMCs

First, we tested the effects of high Pi levels on autophagy in VSMCs. Western blotting was performed to detect the expression of autophagy-related markers in VSMCs exposed to different doses of Pi ( 1 and $3 \mathrm{mM}$ ). We found that treatment with high Pi levels increased LC3II expression in a dose-dependent manner (Fig. 1A, left panel). In addition, results of fluorescence microscopy showed that treatment with high Pi levels promoted autophagic flux in VSMCs in a dose-dependent manner (Fig. 1A, right panel). In contrast, treatment with an autophagy inhibitor decreased LC3II expression (Fig. 1B). These results suggest that treatment with high Pi levels induces autophagy in VSMCs.

\section{MiRNA profiles of VSMCs exposed to high Pi levels}

Accumulating evidence indicates that miRNAs such as miR-18a, miR-21, miR-23a, miR-30b, and miR-31a play critical roles in regulating autophagy [16-19]. In the present study, we examined the expression of the abovementioned miRNAs in VSMCs exposed to high Pi levels.

Fig. 1. Treatment with high Pi levels induces autophagy in VSMCs. VSMCs were cultured with different doses (1 and 3 mM) of Pi. After 24 h, LC3II expression and autophagic flux were measured by performing western blotting (A, left panel) and fluorescence microscopy (A, right panel), respectively. In addition, VSMCs were cultured with Pi and autophagy inhibitors, including 3-methyladenine and Plaquenil, and LC3II expression was determined by performing western blotting (B).

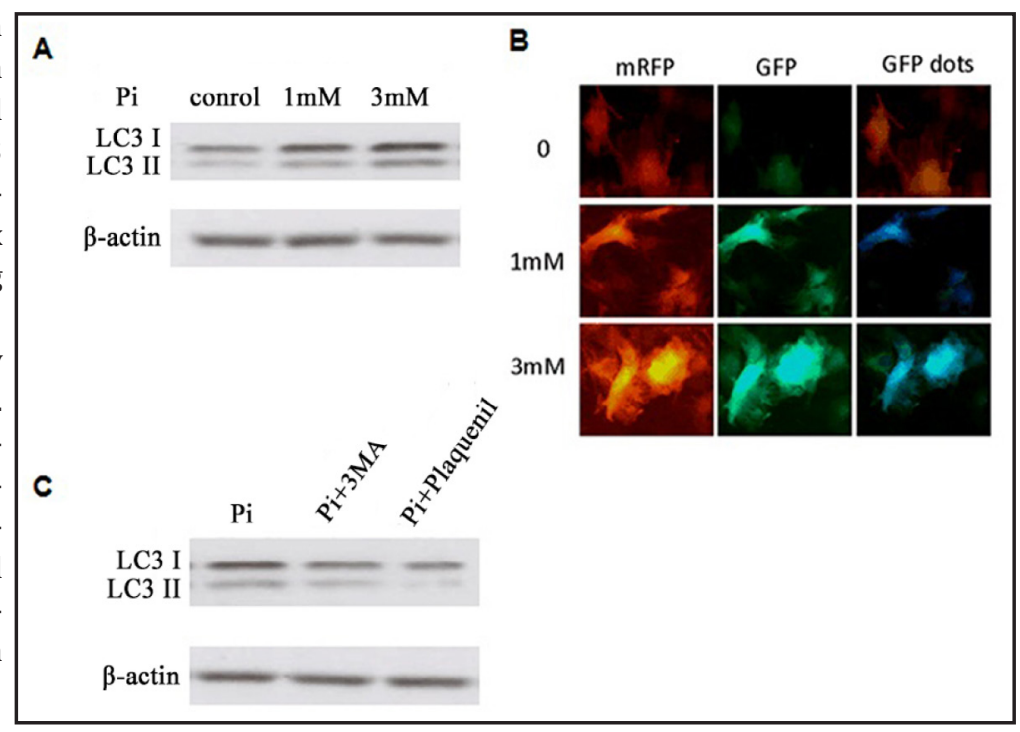

Fig. 2. MiRNA profiles of VSMCs exposed to high Pi levels. PCR was performed to determine the expression profiles of autophagy-related miRNAs, including miR-18a, miR-21, miR23a, miR-30b, and miR-31a, in VSMCs treated with high Pi levels. ${ }^{* *} \mathrm{p}<0.001$.

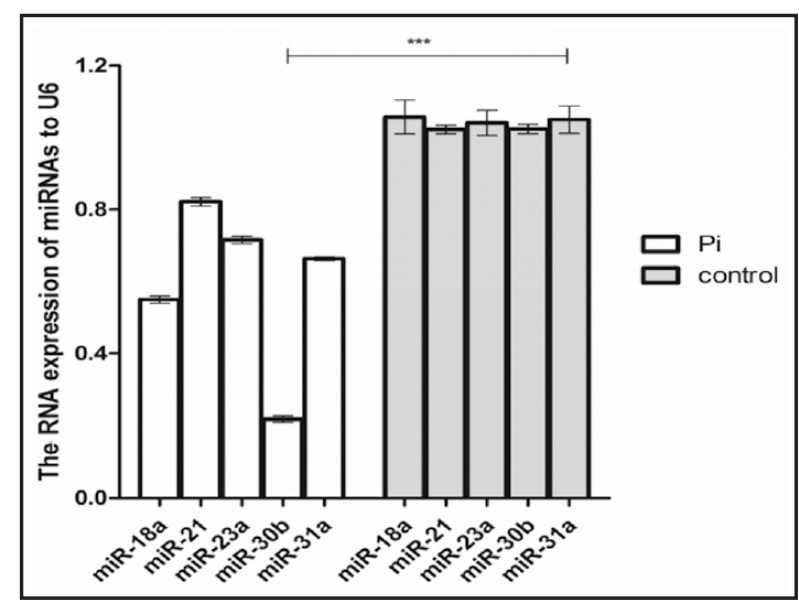



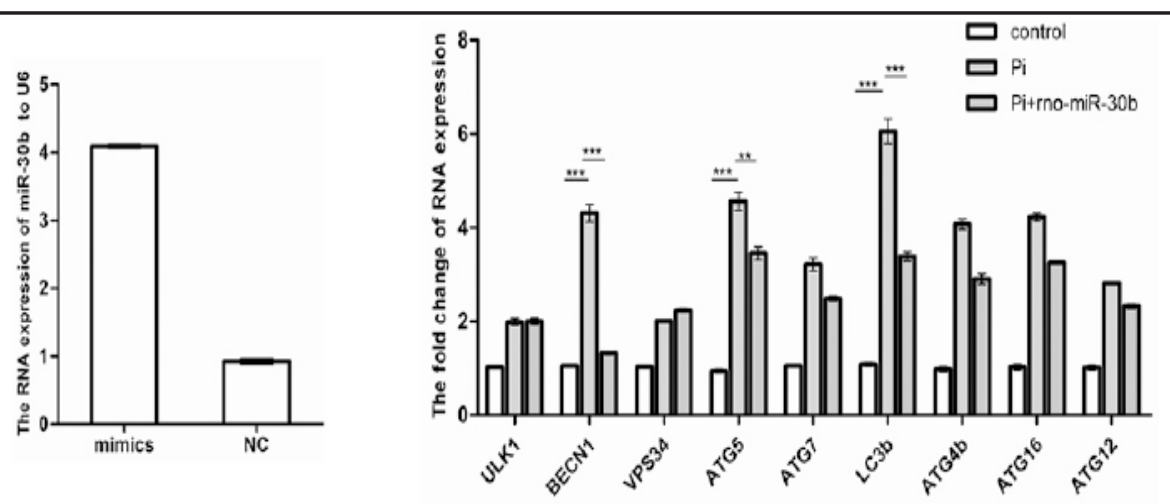

Fig. 3. Upregulation of miR-30b expression inhibits the expression of autophagy-related marker genes. VSMCs were transfected with miR-30b mimics, and real-time PCR was performed to confirm the upregulation of miR-30b expression (left). Moreover, real-time PCR was performed to measure the expression of autophagy-related marker genes, including ULK1, BECN1, VPS34, ATG5, ATG7, LC3b, ATG4b, ATG16, and ATG12, in VSMCs exposed to Pi alone or to a combination of Pi and miR-30b mimics (right). ${ }^{* *} \mathrm{p}<0.01 ;{ }^{* * *} \mathrm{p}<0.001$.

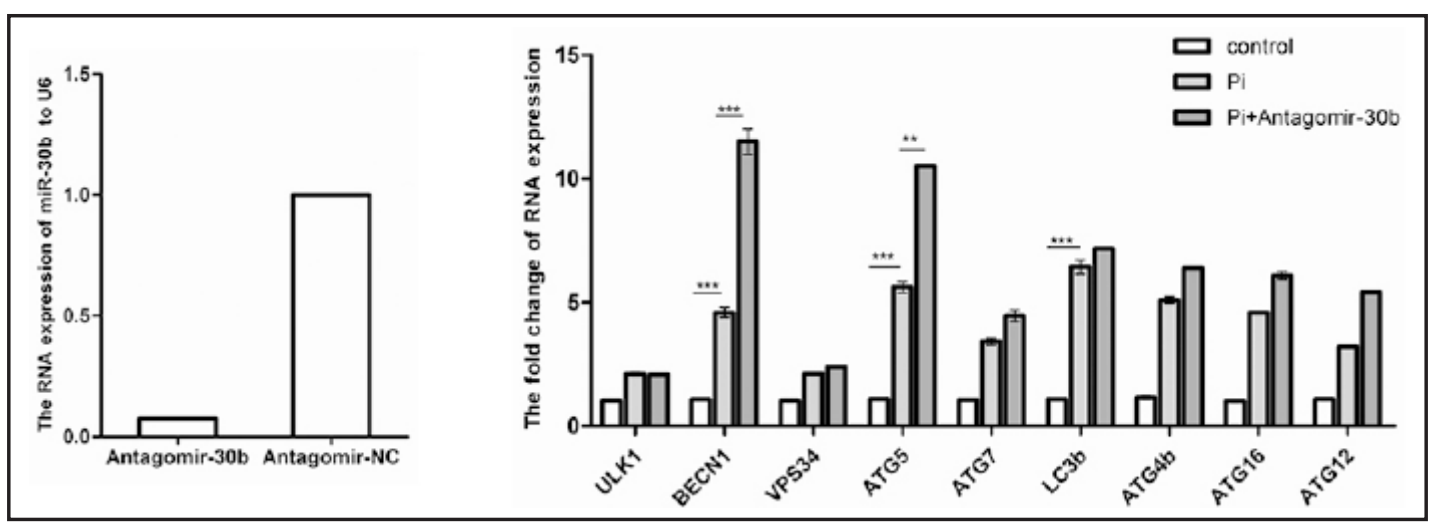

Fig. 4. Downregulation of miR-30b expression promotes the expression of autophagy-related marker genes. VSMCs were transfected with antago-miR-30, and real-time PCR was performed to confirm the downregulation of miR-30b expression (left). In addition, real-time PCR was performed to detect the mRNA expression of autophagy-related marker genes, including ULK1, BECN1, VPS34, ATG5, ATG7, LC3b, ATG4b, ATG16, and $A T G 12$, in VSMCs exposed to Pi alone or to a combination of Pi and antago-miR-30b (right). ${ }^{* *} \mathrm{p}<0.01$; $* * * \mathrm{p}<0.001$.

Fig. 5. miR-30b targets BECN1 in VSMCs. VSMCs were transfected with miR-30b mimics. BECN1 protein expression was measured by performing western blotting (A). Luciferase assay was performed to validate the target (BECN1) of miR-30b $* * \mathrm{p}<0.01$.

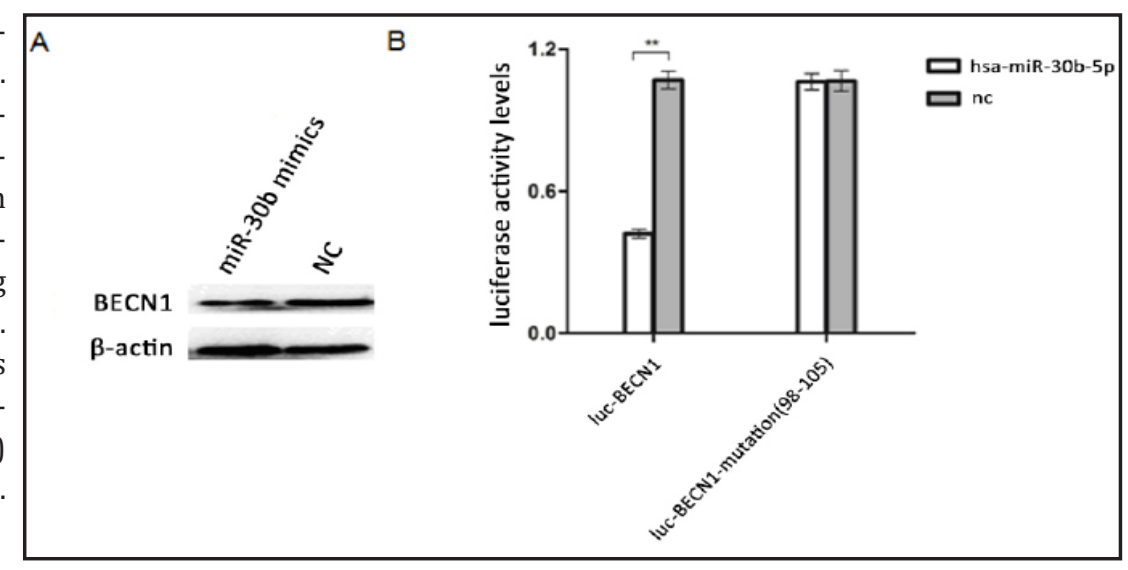

We found that Pi stimulation decreased the expression of miR-18a, miR-21, miR-23a, miR30b, and miR-31a (Fig. 2). Of these, expression of miR-30b was most notably decreased (by approximately five folds); hence, we selected this miRNA for further investigation. 
miR-30b inhibits high Pi level-induced autophagy in VSMCs by targeting BECN1

To understand the role of miR-30b in VSMCs, we transfected these cells with miR-30b mimics. Our results showed that treatment with high Pi levels promoted the expression of autophagy-related marker genes, including BECN1, ATG5, and LC3b. However, upregulation of miR-30b expression inhibited the mRNA expression of these genes in the presence of high Pi levels (Fig. 3), whereas downregulation of miR-30b expression increased the expression of these genes (Fig. 4). Moreover, transfection of VSMCs with miR-30b mimics significantly inhibited BECN1 protein expression (Fig. 5A) and suppressed the luciferase activity of BECN1 (Fig. 5B), suggesting that miR-30b inhibited high Pi level-induced autophagy in VSMCs by targeting BECN1.

\section{Discussion}

Some miRNAs play important roles in autophagy by regulating the key Atg genes [20]. In the present study, we found that high Pi levels induced autophagy and deregulated miRNA expression in VSMCs. Moreover, we found that miR-30b overexpression alleviated high $\mathrm{Pi}$ level-induced autophagy in VSMCs.

Autophagy is an evolutionarily conserved catabolic process involving the formation of autophagosomes that engulf cellular macromolecules and organelles, leading to their breakdown after fusion with lysosomes [4]. Pro-survival functions of autophagy are the most well defined. Autophagy helps cells in dealing with stress by clearing damaged proteins and organelles. However, several recent studies suggest that autophagy is a mechanism of caspase- and apoptosis-independent cell death [7].

A recent study showed that high serum Pi levels are a predictor of chronic kidney disease in patients undergoing homodialysis and peritoneal dialysis [21]. Hyperphosphatemia is an independent risk factor of mortality and myocardial infarction in patients, indicating that high Pi levels play an important role in the pathogenesis of cardiovascular diseases [22]. In the present study, we found that treatment with high Pi levels increased the expression of LC3II, an autophagy-related marker, and promoted autophagic flux in VSMCs. However, treatment with an autophagy inhibitor decreased LC3II expression, suggesting that high $\mathrm{Pi}$ levels induced autophagy in VSMCs.

Accumulating evidences indicate that miRNAs play significant roles in cell behavior, including growth, apoptosis, and autophagy [9-12]. Upregulation of miR-18a expression promotes autophagy in triple-negative cancer cells by suppressing the mTOR signaling pathway [16]. Seca et al showed that inhibition of miR-21 induced autophagy and chemosensitivity in leukemia cells [17]. Another study suggested that miR-23a-regulated autophagy is an important regulator of ultraviolet radiation-induced premature senescence in fibroblasts [18]. In the present study, we evaluated the expression of autophagy-related miRNAs such as miR-18a, miR-21, miR-23a, miR-30b, and miR-31a in VSMCs exposed to high Pi levels. We observed that treatment with high Pi levels decreased the expression of miR-18a, miR-21, miR-23a, miR-30b, and miR-31a. Particularly, treatment with high Pi levels significantly downregulated miR-30b expression. A previous study showed that miR-30b overexpression improved ischemia-reperfusion (I/R) injury in H2C9 cells by downregulating caspase-3 [23]. Similarly, Song et al. found that I/R injury decreased miR-30b expression in rat myocardial cells [24]. Moreover, Zhu et al. observed that miR-30b expression was significantly downregulated in human gastric cancer tissues and that upregulation of miR$30 \mathrm{~b}$ expression promoted the apoptosis of gastric cancer cells [25]. In the present study, we transfected VSMCs with miR-30b mimics and found that upregulation of miR-30b expression inhibited the expression of autophagy-related marker genes (BECN1, ATG5, and $L C 3 b$ ) in the presence of high Pi levels. In contrast, downregulation of miR-30b expression promoted the expression of BECN1 and ATG5, suggesting that miR-30b promoted high Pi level-induced autophagy in VSMCs. Beclin-1, encoded by BECN1, is a mammalian ortholog of yeast Atg6. Beclin-1 interacts with PI3KIII/Vps34 to recruit activators and inhibitors of autophagy to

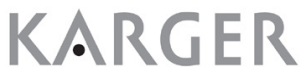




\section{Cellular Physiology Cell Physiol Biochem 2017;42:530-536

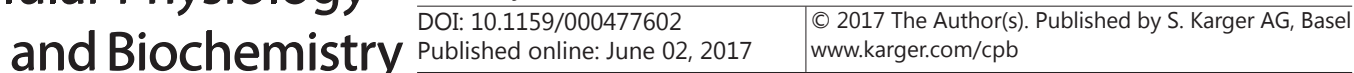

Wang et al.: miR-30b Regulates Autophagy in VSMC via BECN1

finely regulate autophagy. Luciferase activity assay performed in the present study showed that miR-30b directly targeted BECN1 in VSMCs.

In conclusion, results of the present study suggest that treatment with high $\mathrm{Pi}$ levels induces autophagy in VSMCs. Moreover, these results indicate that miR-30b plays an important role in high Pi level-induced autophagy by regulating BECN1 expression, suggesting that miR-30b is a potential target for treating vascular diseases.

\section{Acknowledgements}

This work was supported by a grant from the National Science \& Technology Support Program during the 12th Five-year Plan Period (Grant No. 2011BAI10B02), the Key Social Development Program of Science and Technology Commission of Shenyang of China (Grant No. F16-206-9-04), the Key Social Development Program of Science and Technology Commission of Liaoning Province of China (Grant No. 201404046).

\section{Disclosure Statement}

No conflicts of interest.

\section{References}

1 Legakis JE, Yen WL, Klionsky DJ: A cycling protein complex required for selective autophagy. Autophagy 2007;3:422-432.

-2 Shimobayashi M, Takematsu H, Eiho K, Yamane Y, Kozutsumi Y: Identification of Ypk1 as a novel selective substrate for nitrogen starvation-triggered proteolysis requiring autophagy system and endosomal sorting complex required for transport (ESCRT) machinery components. J Biol Chem 2010;285:36984-36994.

-3 Yang Z, Klionsky DJ: Mammalian autophagy: core molecular machinery and signaling regulation. Curr Opin Cell Biol 2010;22:124-131.

4 Martinet W, De Meyer GR: Autophagy in atherosclerosis: a cell survival and death phenomenon with therapeutic potential. Circ Res 2009;104:304-317.

-5 Yao L, Sun YT, Sun W, Xu TH, Ren C, Fan X, Sun L, Liu LL, Feng JM, Ma JF, Wang LN: High phosphorus level leads to aortic calcification via beta-catenin in chronic kidney disease. Am J Nephrol 2015;41:28-36.

6 Tan M, Yan HB, Li JN, Li WK, Fu YY, Chen W, Zhou Z: Thrombin Stimulated Platelet-Derived Exosomes Inhibit Platelet-Derived Growth Factor Receptor-Beta Expression in Vascular Smooth Muscle Cells. Cell Physiol Biochem 2016;38:2348-2365.

7 Zhu T, Yao Q, Wang W, Yao H, Chao J: iNOS Induces Vascular Endothelial Cell Migration and Apoptosis Via Autophagy in Ischemia/Reperfusion Injury. Cell Physiol Biochem 2016;38:1575-1588.

8 Pestana CR, Oishi JC, Salistre-Araújo HS, Rodrigues GJ: Inhibition of Autophagy by Chloroquine Stimulates Nitric Oxide Production and Protects Endothelial Function during Serum Deprivation. Cell Physiol Biochem 2015;37:1168-1177.

-9 Krol J, Loedige I, Filipowicz W: The widespread regulation of microRNA biogenesis, function and decay. Nat Rev Genet 2010;11:597-610.

10 Louvet L, Metzinger L, Buchel J, Steppan S, Massy ZA: Magnesium Attenuates Phosphate-Induced Deregulation of a MicroRNA Signature and Prevents Modulation of Smad1 and Osterix during the Course of Vascular Calcification. Biomed Res Int 2016;2016:7419524.

11 Zhang T, Tian F, Wang J, Jing J, Zhou SS, Chen YD: Endothelial Cell Autophagy in Atherosclerosis is Regulated by miR-30-Mediated Translational Control of ATG6. Cell Physiol Biochem 2015;37:1369-1378.

12 Su Z, Yang Z, Xu Y, Chen Y, Yu Q: MicroRNAs in apoptosis, autophagy and necroptosis. Oncotarget 2015;6:8474-8490. 


\section{Cellular Physiology Cell Physiol Biochem 2017;42:530-536 \begin{tabular}{l|l} 
DOI: 10.1159/000477602 & Ond 2017 The Author(s). Published by S. Karger AG, Basel \\
www.karger.com/cpb
\end{tabular}}

Wang et al.: miR-30b Regulates Autophagy in VSMC via BECN1

13 Wu H, Wang F, Hu S, Yin C, Li X, Zhao S, Wang J, Yan X: MiR-20a and miR-106b negatively regulate autophagy induced by leucine deprivation via suppression of ULK1 expression in C2C12 myoblasts. Cell Signal 2012;24:2179-2186.

14 Huang Y, Guerrero-Preston R, Ratovitski EA: Phospho-DeltaNp63alpha-dependent regulation of autophagic signaling through transcription and micro-RNA modulation. Cell Cycle 2012;11:1247-1259.

-15 Ciceri P, Volpi E, Brenna I, Arnaboldi L, Neri L, Brancaccio D, Cozzolino M: Combined effects of ascorbic acid and phosphate on rat VSMC osteoblastic differentiation. Nephrol Dial Transplant 2012;27:122-127.

-16 Fan YX, Dai YZ, Wang XL, Ren YQ, Han JJ, Zhang H: MiR-18a upregulation enhances autophagy in triple negative cancer cells via inhibiting mTOR signaling pathway. Eur Rev Med Pharmacol Sci 2016;20:21942200.

17 Seca H, Lima RT, Lopes-Rodrigues V, Guimaraes JE, Almeida GM, Vasconcelos MH: Targeting miR-21 induces autophagy and chemosensitivity of leukemia cells. Curr Drug Targets 2013;14:1135-1143.

- 18 Zhang JA, Zhou BR, Xu Y, Chen X, Liu J, Gozali M, Wu D, Yin ZQ, Luo D: MiR-23a-depressed autophagy is a participant in PUVA- and UVB-induced premature senescence. Oncotarget 2016;7:37420-37435.

19 Tang B, Li N, Gu J, Zhuang Y, Li Q, Wang HG, Fang Y, Yu B, Zhang JY, Xie QH, Chen L, Jiang XJ, Xiao B, Zou QM, Mao XH: Compromised autophagy by MIR30B benefits the intracellular survival of Helicobacter pylori. Autophagy 2012;8:1045-1057.

20 Frankel LB, Lund AH: MicroRNA regulation of autophagy. Carcinogenesis 2012;33:2018-2025.

21 Kestenbaum B: Mineral metabolism disorders in chronic kidney disease. JAMA 2011;305:1138-1139.

22 Kestenbaum B, Sampson JN, Rudser KD, Patterson DJ, Seliger SL, Young B, Sherrard DJ, Andress DL: Serum phosphate levels and mortality risk among people with chronic kidney disease. J Am Soc Nephrol 2005;16:520-528.

23 Li T, Sun ZL, Xie QY: Protective effect of microRNA-30b on hypoxia/reoxygenation-induced apoptosis in H9C2 cardiomyocytes. Gene 2015;561:268-275.

-24 Song CL, Liu B, Wang JP, Zhang BL, Zhang JC, Zhao LY, Shi YF, Li YX, Wang G, Diao HY, Li Q, Xue X, Wu JD, Liu J, Yu YP, Cai D, Liu ZX: Anti-apoptotic effect of microRNA-30b in early phase of rat myocardial ischemiareperfusion injury model. J Cell Biochem 2015;116:2610-2619.

-25 Zhu ED, Li N, Li BS, Li W, Zhang WJ, Mao XH, Guo G, Zou QM, Xiao B: miR-30b, down-regulated in gastric cancer, promotes apoptosis and suppresses tumor growth by targeting plasminogen activator inhibitor-1. PLoS One 2014;9:e106049. 